\title{
Multivariate curve resolution for infrared spectrometric monitoring of the microwave assisted synthesis of bio-based polyols
}

\author{
Mir Mohammad Alavi Nikje* , Mohammadreza Khanmohammadi, Fahimeh Abedinifar, Amir \\ Bagheri Garmarudi
}

\begin{tabular}{l}
\hline A R T I C L E I N F O \\
\hline Article history: \\
Received March 30, 2012 \\
Received in Revised form \\
April 7, 2012 \\
Accepted 8 April 2012 \\
Available online \\
8 April 2012 \\
\hline Keywords: \\
Infrared spectrometric \\
Epoxidized soybean oil \\
Microwave irradiation \\
Chemometrics \\
\hline
\end{tabular}

A B S T R A C T

A new analytical approach was proposed to monitor the reaction between epoxidized soybean oil and diethylene glycol in the presence of $\mathrm{ZnCl} 2$ as an epoxide ring opening catalyst for microwave assisted synthesis of polyol. Infrared spectra of the products were used as the input data, being processed by multivariate curve resolution - alternating least squares (MCR-ALS) for quantitative monitoring. The data obtained in different MW powers were analyzed by MCR factor analysis coupled with ALS optimization procedure. Standard deviation of residuals for experimental data and the variance explained at the optimum condition (r2) were 0.135 and $97.24 \%$ respectively.

(C) 2012 Growing Science Ltd. All rights reserved.

\section{Introduction}

Nowadays, biodegradable polyols are of high interest in polyurethane industry. There are several bio-based polyols, synthesized from epoxidized soybean oil (ESBO) ${ }^{1}$. Most of the previous reports in this filed have focused on a ring opening process, by ESBO using conventional heating. Several catalysts or nucleophile reagents have been employed ${ }^{2-7}$. Recently, MW irradiation has been introduced as a green power source, which provides several advantages while a chemical process is conducted. Some of them are: acceleration in reaction rate, benefiting mild reaction conditions, achieving higher yields in very short times and possibility for omitting the solvent from reaction system. Thus, MW processing is environmentally clean and makes the conditions controllable ${ }^{8}$. One of the main milestones in chemical reactions is to determine the reaction yield what is related to the 
energy consumption, reaction times and process cost balances which all are effective while a process is scaled up to be industrially performed. Several analytical methods have been introduced for reaction monitoring and yield analysis for industrial and commercial purposes. Obviously, most of the chromatographic approaches are time consuming and require several chemical reagents and solvents which would increase the costs of analysis besides being harmful to the environment. This would effectively castrate the "Green Chemistry" aims which have been proposed. In the other hand, FTIR spectrometry is a fast analytical technique which provides useful qualitative and quantitative information. It would simplify sample handling and avoid common operating problems. Thus the application of infrared spectrometric methods is dramatically increased for different goals. It is also mentionable that nowadays, every analytical technique is coupled with a chemometrics technique to improve the capabilities, performing more reliable models and achieving more precise results. Multivariate Curve Resolution Alternating Least Square (MCR-ALS) ${ }^{9}$ is a chemometrics method which has been applied in different analytical aspects. It has introduced chemometrics to be used for the resolution of multiple component responses in unknown unresolved mixtures. Essentially, any multi component system that gives data tables or data matrices as result which is describable by a bilinear model is possible to be processed by MCR-ALS. This description includes all kinds of processes and mixtures (e.g., chemical reactions, industrial processes, spectroscopic images or environmental data) monitored by diverse multivariate responses, such as spectroscopic measurements. In the present study, infrared spectrometric data from the product of MW assisted alcoholysis of ESBO under solvent-free condition in the presence of $\mathrm{ZnCl}_{2}$ has been treated by MCRALS to determine the optimized reaction yield (Fig. 1).

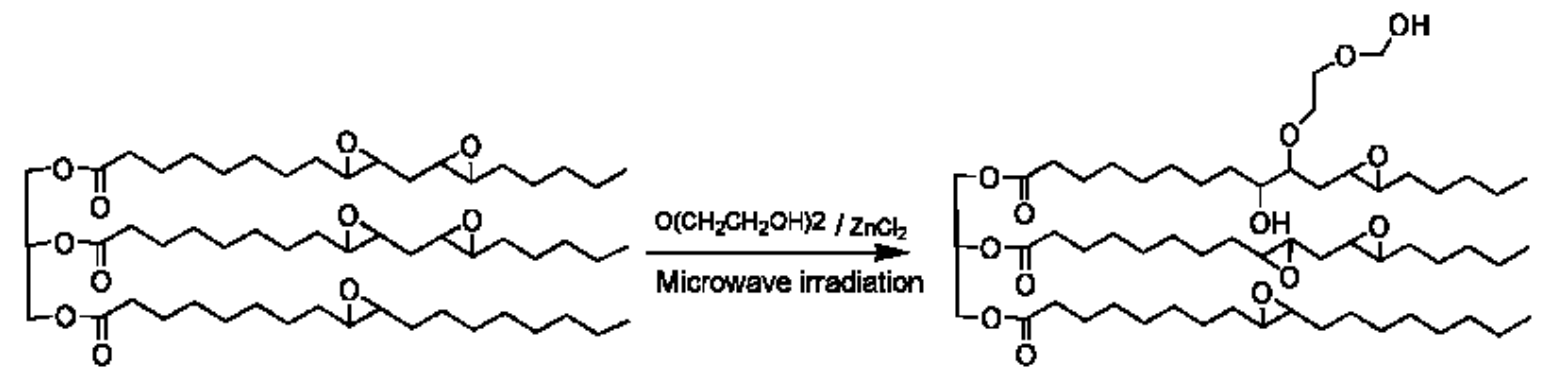

Fig. 1. Reaction scheme for ring opening of epoxidized Soybean Oil under MW irradidtio 2

\section{Experimental}

\subsection{Materials and apparatus}

ESBO (MW: 865gr/mol, O.N:6.02\%, Iodine value: 4) was Baspar Company (Tehran, Iran). Diethylene glycol (DEG) and zinc chloride were of analytical grade from Merck (Darmstadt, Germany). All chemicals were used as received without any further pretreatment or purification. A Milestone Microsynth "NP" Ethos 1600 microwave oven at atmospheric pressure was used for conduction of all reactions. Infrared spectra were recorded by using a Tensor 27 BRUKER spectrometer. ${ }^{1} \mathrm{HNMR}$ and ${ }^{13} \mathrm{CNMR}$ spectra were done using a BRUKER CRX 300 instrument using $\mathrm{CDCl}_{3}$ as a solvent and tetramethylsilane as an internal standard. The obtained spectrometric data was processed by multivariate curve resolution alternating least square (MCR-ALS). Using MATLAB ${ }^{\circledR}$ ver. 7.4 (Mathwork Co., USA) software, the useful chemical information was extracted from FTIR spectra.

\subsection{Brief description of the performed epoxide ring opening reactions}

The mixture of ESBO (4.325 g, $5 \mathrm{mmol})$, DEG (3.18 g, $30 \mathrm{mmol})$ and $\mathrm{ZnCl}_{2}(5-10 \%)$ was prepared in a three necked microwave glassy reactor equipped with a condenser, a thermal sensor and a magnetic stirrer, being irradiated at $100{ }^{\circ} \mathrm{C}$ for 10 minutes. In the first step, and in order to optimize the effect of catalyst content in process yield, a series of reactants mixture was prepared containing 5 - 
$10 \% \mathrm{w}$ of $\mathrm{ZnCl}_{2}$. The reaction was conducted at $500 \mathrm{~W}$ microwave power, while the ramp time and hold time were 2 and 8 min respectively. In order to remove the probable un-reacted DEG and $\mathrm{ZnCl}_{2}$ the product was washed with distilled water and extracted by diethyl ether. In the next step, a rotary evaporator was used to remove the excess diethyl ether. Finally, remained oil was characterized by spectrometric methods. The ${ }^{1}$ HNMR spectra of purified products were used to determine the reaction yield. All reactions were evaluated according to the variations in peak area, evolutionary slight appearance or disappearances of some significant signals in ${ }^{1} \mathrm{HNMR}$ spectra. The effect of microwave power was investigated for a series of reactions, containing optimized amount of $\mathrm{ZnCl}_{2}$ and FTIR spectra were recorded to be processed for monitoring aims.

\subsection{Quantitative monitoring of the ring opening reaction}

The main variations in the ${ }^{1} \mathrm{HNMR}$ spectra during the reaction progress are located at $2.7-3.0 \mathrm{ppm}$ (epoxy ring attached protons) and 3.5-4.0 ppm (protons near $-\mathrm{OH}$ groups) spectral regions. The first region's intensity is decreased and the second one's increased during the reaction. It is mentionable that other ${ }^{1} \mathrm{HNMR}$ signals of ESBO and DEG containing ring opened ESBO are similar. The reaction yield was determined according to the following equation:

$\left(\%\right.$ Yield $\left.=\frac{A_{2.7-3.0}(\text { polyol })}{A_{2.7-3.0}(E S B O)} \times 100\right)$

As mentioned previously, the FTIR spectra of reactions were obtained to be processed by MCR-ALS technique.

\section{Results and Discussion}

\subsection{Optimizing the catalyst content}

As mentioned before, $\mathrm{ZnCl}_{2}$ was added to the reaction system, $5-10 \mathrm{w} \%$ and the reaction was performed at $500 \mathrm{~W}$ power of $\mathrm{MW}$ reactor in $10 \mathrm{~min}$. The obtained from ${ }^{1} \mathrm{HNMR}$ spectra demonstrated that the system containing $9 \% \mathrm{w}$ and more of $\mathrm{ZnCl}_{2}$ would provide the maximum yield. The obtained results are shown in Fig. 2. In order to ensure the adequate amount of catalyst, all reaction systems were prepared by $10 \% \mathrm{w}$ of $\mathrm{ZnCl}_{2}$.

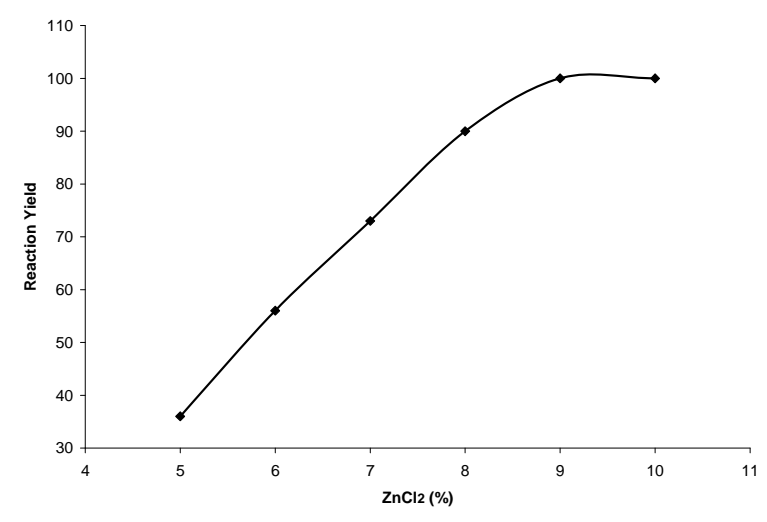

Fig. 2. Effect of catalyst loading on reaction yield

\subsection{Data processing for reaction monitoring}

The studied chemical reaction it the present work is an evolving system due to variations in the quantity of reactants. These variations would affect the FTIR signals' intensity. It is of high interest to use a method for data processing which could usefully determine the reaction yield. Suitable approaches for such a system are mainly based on curve resolution algorithms to the data matrix 
obtained from them. In the first step, principal component analysis (PCA) is performed to examine the data. PCA is a widely accepted method that reduces complex spectral data into much fewer dimensions using principal components (eigenvectors) and scores. PCA is performed to estimate the possible number of components. It is assumed that a bilinear relation exists between experimental data, concentrations and spectra of the components of analogues structure to the generalized law of Lambert-Beer. PCA creates "new" dimensions of the data and evaluates a reduced number of independent factors or principal components describing the information included in a system of characteristic but partly dependent variables. The aim of PCA is to find a few components which explain the major variations within the data matrix. The obtained data set from FTIR spectrometry can be described as a sum of responses for each principal component in the data, which are characterized as:

$\boldsymbol{X}=\boldsymbol{C} . \boldsymbol{S}+\boldsymbol{E}$

where $\boldsymbol{X}$ is the original spectral data matrix $\boldsymbol{C}$ is score matrix, $\boldsymbol{S}$ is the loading matrix and $\boldsymbol{E}$ is an error matrix. One of the most critical parameters, which play an important role in the application of curve resolution technique, is the determination of the number of factors or components that cause the variability in the data set ${ }^{10}$.

The main question which is going to be answered by MCR is the number of species causing the observed data variance and their related spectral and concentration profile for a given data matrix. This is possible if it is assumed that the rank of the data matrix is equal to the number of active components and there are no interfering parameters e.g. noise. In case of a reaction monitoring process, the probable side reactions would cause some difficulties in determination of the real number of PCs. If one of the concentration or spectral profile's matrices is full rank, the rank of the other matrix is useful to analyze the rank of data matrix. As mentioned before, the present study was of an evolving system and the data sets are often rank deficient. A main cause of rank deficiency is the socalled rank overlap, which appears when various chemical species have common profiles in one of the two orders of measurement. When rank overlap is present in the concentration profiles, the rank of data matrix is equal to the number of independent concentration profiles. The studied system may be rank-deficient, due to the presence of species with similar characteristics to the analyte. Thus it is decided to use augmented matrices. In any case, the success of rank augmentation is linked with the suitable full rank information appended to the rank-deficient matrix. Then evolving factor analysis (EFA) was applied to estimate the pure spectra and ALS optimization program was utilized to predict the reaction yield ${ }^{11,12}$.

\section{Multivariate curve resolution alternating least squares (MCR-ALS)}

MCR-ALS is considered a general purpose factor analysis technique which is also applicable for different aims. MCR methods are based on a bilinear model such as:

$\mathbf{D}=\mathbf{C S}^{\mathrm{T}}+\mathbf{E}$

MCR-ALS decomposes the matrix D (data matrix) bilinearly into the pure response profiles which are related to variation of each contribution in the row and the column directions, represented by matrices $\mathbf{C}$ and $\mathbf{S}^{\mathrm{T}}$, respectively, which are responsible for the observed data variance. $\mathbf{E}$ is the matrix of residuals not explained by the model and ideally should be close to the experimental error. In the present study, rows of matrix $\mathbf{D}$ are the spectra recorded during the experimental step, the column profiles of matrix $\mathbf{C}$ and the row profiles of $\mathbf{S}^{\mathbf{T}}$ are usually associated respectively with the concentration and pure spectral profiles of the resolved components which are obtained by ALS. The obtained results must optimally fit the experimental data matrix $(\mathbf{D})^{13}$. This optimization is carried out using initial estimates of either $\mathbf{C}$ or $\mathbf{S}^{\mathrm{T}}$ by some routes derived from SIMPLISMA or evolving factor analysis, applying constraints to model the shapes of the profiles ${ }^{14}$. Fig. 3 shows the concentration and spectral profile for reaction product. 

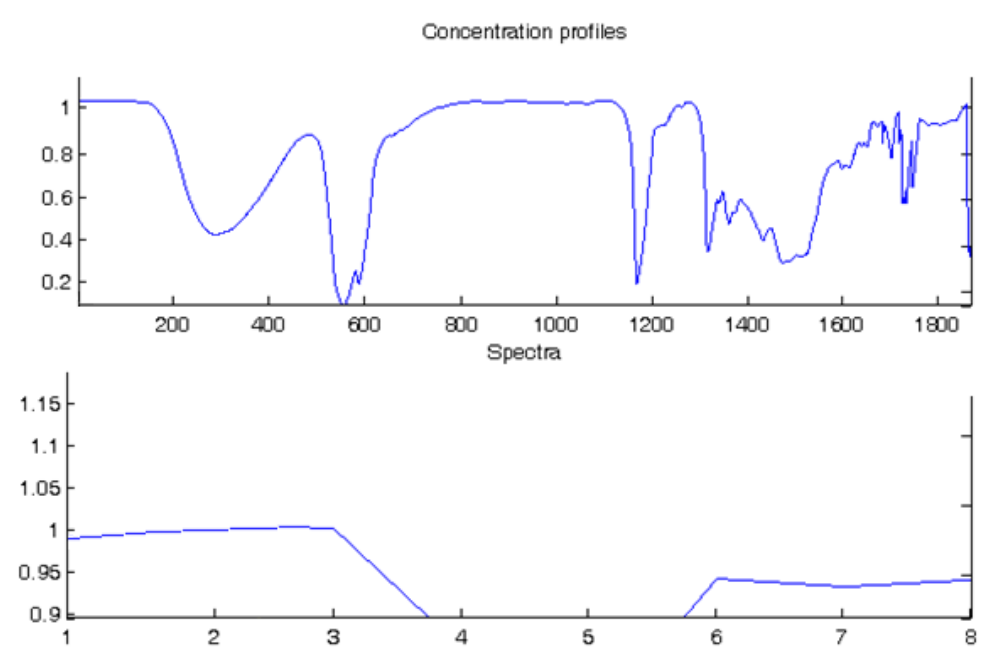

Fig. 3. Spectral and concentration profile of MW effect of reaction yield

\subsection{MCR-ALS processing of FTIR data}

The FTIR spectra of the reaction product in different conditions were used in MCR-ALS approach. The individual spectra obtained in different reaction conditions would form the rows of data matrix and the absorbance measured values at each wavenumber are in the column. This constraint implies the establishment of calibration models for MCR-ALS, to be used for the quantitative determination of the reaction yield. As mentioned before, the reaction system which contains $10 \%$ of $\mathrm{ZnCl} 2$ would provide the max yield and is free of reactants. The comparison between FTIR spectra demonstrated that epoxide ring opening would increase the hydroxyl group's signal at $3420 \mathrm{~cm}^{-1}$, while the epoxide group's signal at $830 \mathrm{~cm}^{-1}$ is disappeared. An advantage of MCR-ALS would be its ability in calculation of pure spectra of all chemical species in the solution. Once the concentration profile of the absorbing species coexisted in the solution system was obtained, the pure spectra of these components can be easily calculated by pre-multiplication of experimental absorbance data matrix with the pseudo-inverse of concentration profile data matrix. The optimized models were applied for validation and quantitative determination of reaction yield. The standard deviation of residuals for experimental data was 0.135 and the variance explained at the optimum condition $\left(\mathrm{r}^{2}\right)$ was $97.24 \%$. Fitting error or lack of fit was calculated as:

$$
\left.\operatorname{LOF}(\%)=\sqrt{d^{*}}{ }_{i j}-d_{i j}\right) / \sum d^{2}{ }_{i j} \times 100
$$

which was 2.18 and 6.16 for PCA and experimental data respectively. The results obtained by MCRALS treatment of FTIR spectra from reactions with different MW power are shown in Fig. 4. It is observed that MW power would straightly affect the reaction yield.

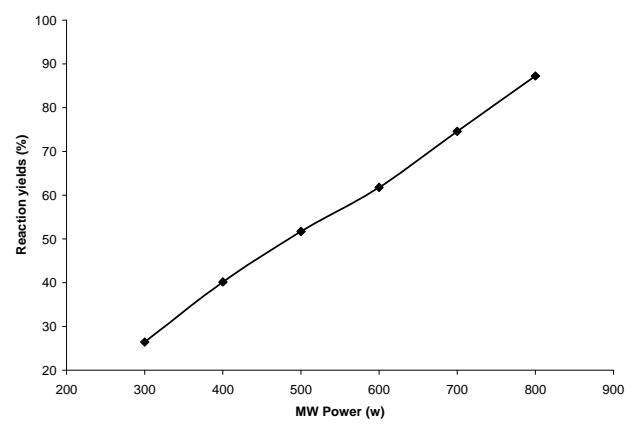

Fig. 4. Effect of MW power on reaction yield 


\section{Conclusion}

MCR-ALS chemometric technique was applied in yield determination for a ring opening reaction of ESBO under MW irradiation and solvent-free condition. The disappearance of epoxy groups in the FTIR spectra and the emergence of the hydroxyl groups were the main used input data. It was observed that in MW irradiation case, the ring opening reaction will take place milder and probability of transesterification side reaction has been decreased at the comparison with the conventional heating method.

\section{References}

1. Gue, A., Zhang, W., Petrovic, S., Z. (2006). Structure-Property Relationships in Polyurethanes Derived from Soybean Oil. J. Mater. Sci., 41, 4914-4920.

2. Gue, Y., Hardesty, J., H., Mannari, V., M., Massingill, J., L. (2007). Hydrolysis of Epoxidized Soy Oil in the Presence of Phosphoric Acid. J. Am. Oil Chem. Soc., 84, 929-935.

3. Petrovic, Z., S., Javni, I., Gue, A., Zhang, W. (2002). Method of Making Natural Oil-based Polyols and Polyurethanes Therefrom. U. S. Patent 6,433,121.

4. Campanella, A., Baltanas, M., A. (2005). Degradation of Oxiran of Epoxidized Vegetable Oil in Liquid-Liquid System: I. Hydrolysis and Attack by $\mathrm{H}_{2} \mathrm{O}_{2}$. Latin Am. Appl. Res., 3, 205-210.

5. Petrovic, Z., S., Guo, A., Zhang, W. (2000). Structure and Properties of Polyurethane Based Halogenated and Nonhalogenated Soy-polyols. J. Polym. Sci. Pol. Chem., 38, 4062-4069.

6. Meffert, A., Kluth, H. (1989). Process for the Preparation of Modified Triglycerides. U. S. Patent $4,886,893$.

7. Ionescu, M., Petrovic, Z., S., Wan, X. (2007). Ethoxylated Soybean Polyols for Polyurethanes. J. Environ. Polym. Degr., 15, 237-243.

8. Kappe, C., O. (2004). Controlled Microwave Heating in Modern Organic Synthesis. Angew. Chem. Int. Ed., 43, 6250-6284.

9. Jaumot, J., Gargallo, R., de Juan, A., Tauler, R.(2005). A Graphical User-friendly Interface for MCR-ALS: a New Tool for Multivariate Curve Resolution in MATLAB. Chemometr. Intell. Lab., 76, 101-110.

10. de Juan, A., Dysson, R., Marcolli, C., Rault, M., Tauler, R., Maeder, M. (2004). Spectroscopic Imaging and Chemometrics: a Powerful Combination for Global and Local Sample Analysis. Trends Anal. Chem., 23, 70-79.

11. Azzouz, T., Tauler, R. (2008). Application of Multivariate Curve Resolution Alternating Least Squares (MCR-ALS) to the Quantitative Analysis of Pharmaceutical and Agricultural Samples. Talanta, 74, 1201-1210.

12. Berbel, F., Kapoya, E., Diaz-Cruz, J., M., Arino, C., Esteban, M., Tauler, R. (2003). Multivariate Resolution of Coeluted Peaks in Hyphenated Liquid Chromatography - Linear Sweep Voltammetry. Electroanal., 15, 499-508.

13. Jaumot, J., Gargallo, R., Escaja, N., Gonzalez, C., Pedroso, E., Tauler, R. (2002). Multivariate Curve Resolution: a Powerful Tool for the Analysis of Conformational Transitions in Nucleic Acids. Nucleic Acid Res., 30 (17), e92/1-e92/18.

14. Lopez, M., J., Arino, C., Diaz-Cruz, S., Diaz-Cruz, J., M., Tauler, R., Esteban, M. (2003). Voltammetry Assisted by Multivariate Analysis as a Tool for Speciation of Metallothioneins: Competitive Complexation of $\alpha$ - and $\beta$-Metallothionein Domains with Cadmium and Zinc. Environ. Sci. Technol., 37, 5609- 5616. 